\title{
On the phenomenon of vortex street breakdown
}

\author{
By WILLIAM W. DURGIN† \\ AND STURE K. F. KARLSSON \\ Division of Engineering, Brown University, Providence, R.I. 02912
}

(Received 6 October 1970)

A von Kármán vortex street generated in the usual way was subjected to a deceleration, thereby changing the ratio of longitudinal to lateral spacing between the vortices. Distortion of the individual vortices followed which resulted in annihilation of concentrated vortex regions and creation of a stationary wake flow. This wake flow was itself dynamically unstable and developed into a new vortex street of a different frequency from the initial one. The breakdown of the initial vortex street is qualitatively explained by considering the convection of a concentrated vortex region due to the motion imposed by all the other vortices.

\section{Introduction}

For nearly a century the von Kármán vortex street has been the subject of many experimental and theoretical investigations. Seemingly basic to the study of time-dependent flows because of its simple periodicity, the vortex street has, nevertheless, evaded all but the most rudimentary of mathematical descriptions. Being relatively unguided by theory, experimentalists have, for the most part, always made the same type of measurements, namely of velocity and geometry in a plane through the vortex filaments.

Classically the vortex street has been divided into three regions (cf. Schaefer \& Eskinazi 1959): the formation, a stable, and an unstable region. The formation region has been synthesized utilizing numerical integrations on a digital computer (Payne 1958). The stable region was originally described by von Kármán (von Kármán \& Rubach 1912) and more recently by Schaefer \& Eskinazi. The unstable region was thought to precede turbulence or accomplish the viscous annihilation of the vortex street.

In 1959 Taneda found an alternative development for the vortex street. $\mathrm{He}$ discovered that far downstream the original vortex street disappeared and a new one of larger scale appeared. That he was the first to observe this phenomenon is not surprising since his apparatus was unique in being able to preserve the vortex trail for long distances. Credibility was lent to Taneda's observations by Zdravkovich (1968) who photographed the vortex trails of three cylinders in close proximity. For certain spacings of the cylinders the centre four vortex rows

$\uparrow$ Present address: Department of Engineering Science and Mechanics, University of Florida, Gainesville, Florida 32601. 
annihilated each other leaving the two outside rows which quickly changed to the lower frequency, larger scale vortex street observed by Taneda. Still another situation which produces essentially the same phenomenon was observed by Karlsson (unpublished report). In this case the vortices shed from a circular cylinder are convected into the region of deceleration in front of a larger circular cylinder placed perpendicular to the first cylinder and the flow. Somewhere in the region between the cylinders a similar change of scale is observed. The present study utilizes this geometry.

\section{Experimental apparatus and instrumentation}

Two different low-speed wind tunnels were used in the course of the experimental work, both having rectangular cross-section test sections measuring $10 \mathrm{in}$. by $15 \mathrm{in}$. and $22 \mathrm{in}$. by $32 \mathrm{in}$. respectively. These tunnels have recently been described by Sadeh, Sutera \& Maeder (1968) and Wood (1969).

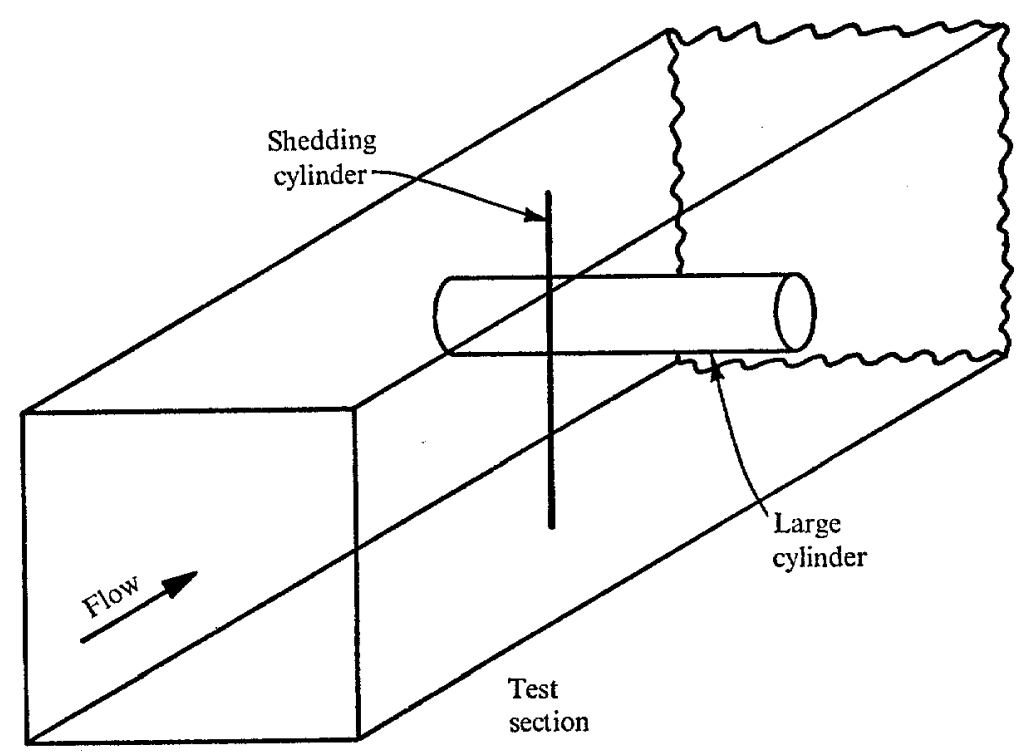

Figure 1. Geometrical arrangement in test section.

In the smaller of the two wind tunnels we studied a vortex street approaching the forward stagnation point of a $1.5 \mathrm{in}$. diameter circular cylinder, and the corresponding large cylinder in the large wind tunnel was $4.5 \mathrm{in}$. in diameter. Figure 1 illustrates the experimental arrangement.

All velocity measurements were made using the anemometer systems described in Sadeh et al. and Wood operated in the constant-temperature mode. Preliminary experiments showed that a probe with long thin support needles oriented across-stream was the only type which did not affect the flow field of interest significantly and was used exclusively in the final measurements.
One hot wire was supported on a test section, which allowed continuc co-ordinate axes. The hot wire wa cylinder. A two-dimensional lathe co on the opposite side of the test sect

\section{The flow field}

The flow in the wake of the sheddi regions, each of which will be discu were made in the plane of symmetr.

Within a range of free-stream $v$ shedding cylinder exhibited a perioe single frequency sinusoidal wave $\mathrm{w}$ velocity fluctuations due to convect probe was moved in towards the fluctuations had a dominant second forms were characteristic of the vor wake flow behind a cylinder appro and 300 and were extensively studie However, as the probe was moved finally becoming undetectable. We calm region. Here no velocity fluc however, showed a large deficit acro downstream was characteristically a at least.

The end of the calm region was instability and the formation of a $n$ wavelength than the original. Figur as a function of time in this wake. For fluctuations in the original vortex st downstream, but outside the boun teristically $0.03 \mathrm{in}$. thick), this secor low frequency fluctuations.

A wave analyzer was used to deter signal, and the fundamental was ac frequency standard. Second and $t$ especially prominant immediately be

A comparison between the two sim in figure 3 in which the non-dimensio against the non-dimensional second shedding cylinder diameter and $\nu$ the some of the data is from the small $n$ turbulence level than the large tunne 
One hot wire was supported on a traversing device, mounted external to the test section, which allowed continuous displacement along each one of the three co-ordinate axes. The hot wire was always oriented parallel to the shedding cylinder. A two-dimensional lathe compound (oriented horizontally) was mounted on the opposite side of the test section to hold the reference hot-wire probe.

\section{The flow field}

The flow in the wake of the shedding cylinder can be divided into three distinct regions, each of which will be discussed separately. The following observations were made in the plane of symmetry of the flow around the large cylinder.

Within a range of free-stream velocities the wake immediately behind the shedding cylinder exhibited a periodic structure. Near the outside of the wake a single frequency sinusoidal wave was observed on the oscilloscope representing velocity fluctuations due to convected vortices on that side of the wake. As the probe was moved in towards the centre of the wake the measured velocity fluctuations had a dominant second harmonic component. The observed waveforms were characteristic of the von Kármán vortex street which exists in free wake flow behind a cylinder approximately between Reynolds numbers of 50 and 300 and were extensively studied by Kovasznay (1949) and Roshko (1953). However, as the probe was moved downstream, the fluctuations grew smaller, finally becoming undetectable. We have called this region of the flow field the calm region. Here no velocity fluctuations were visible. The mean velocity, however, showed a large deficit across the wake. The length of this calm region downstream was characteristically an inch at most and practically non-existent at least.

The end of the calm region was signalled by the development of a growing instability and the formation of a new vortex street of much larger width and wavelength than the original. Figure 2 (plate 1) shows the velocity fluctuations as a function of time in this wake. For reference the upper trace shows the velocity fluctuations in the original vortex street close to the shedding cylinder. Further downstream, but outside the boundary layer of the large cylinder (characteristically $0.03 \mathrm{in}$. thick), this second vortex street disappeared, leaving some low frequency fluctuations.

A wave analyzer was used to determine the frequency content of the hot-wire signal, and the fundamental was acourately determined by comparison with a frequency standard. Second and third harmonics of the fundamental were especially prominant immediately behind the shedding cylinder.

A comparison between the two simultaneously existing vortex streets is shown in figure 3 in which the non-dimensional primary street frequency $f d^{2} / \nu$ is plotted against the non-dimensional secondary street frequency $\sigma d^{2} / \nu$ where $d$ is the shedding cylinder diameter and $\nu$ the kinematic viscosity. As noted on the figure some of the data is from the small wind tunnel which has a higher free-stream turbulence level than the large tunnel.

ing the anemometer systems de1 the constant-temperature mode. be with long thin support needles ich did not affect the flow field of $y$ in the final measurements. 


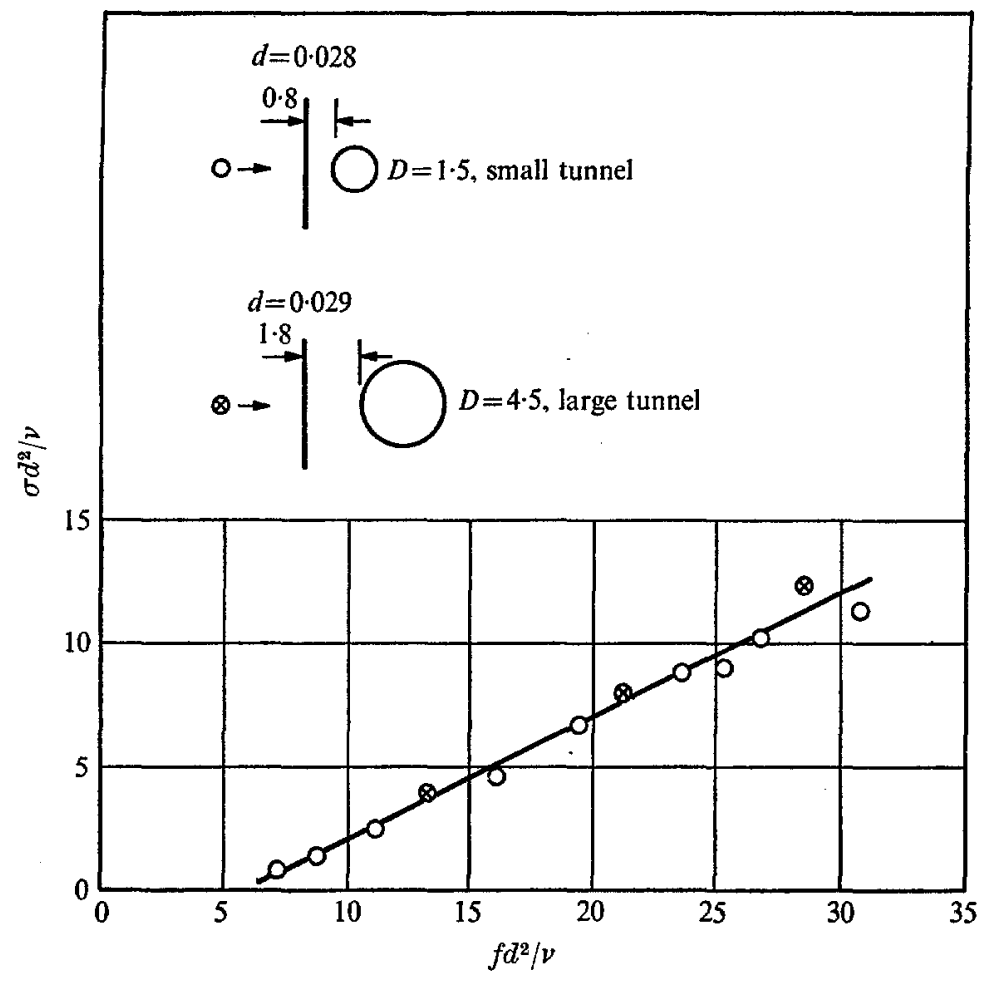

Figure 3. Secondary wake frequency $v s$. primary wake frequency.

\section{Velocity profiles}

The wake profile measurements were made in the large wind tunnel, thus the large cylinder had a diameter of $4.5 \mathrm{in}$. The shedding cylinder was $0.029 \mathrm{in}$. in diameter and the distance between the cylinders was $1.8 \mathrm{in}$. The free-stream velocity was $15.9 \mathrm{ft} / \mathrm{sec}$, the shedding frequency $700 \mathrm{~Hz}$, and the air temperature $75^{\circ} \mathrm{F}$.

The mean velocity $U$ and the normalized r.m.s. fluctuations of the velocity, $\left(\left(\overline{u^{2}}\right)^{\frac{1}{2}} / U\right)$, were measured in the plane of symmetry of the large cylinder using standard hot-wire techniques. Velocity measurements were made every 0.001 in. in the cross-wake $(y)$ direction and every $0.05 \mathrm{in}$. in the downstream $(x)$ direction in the primary vortex street, increasing to every $0.1 \mathrm{in}$. in the calm and secondary street regions. The wake was found to be symmetrical, therefore, the measurements were limited to one side.

Figure 4 shows the mean velocity plotted against $y$ for each downstream station. The heavy curve at $x=0.85 \mathrm{in}$. marks the disappearance of the primary vortex street as indicated by the fluctuation measurements. The second heavy curve at $x=1.25 \mathrm{in}$. marks the appearance of the secondary vortex street. These profiles can be seen to drop from some value outside the wake to a minimum on the wake centreline where the slope must be zero if the wake is symmetrical. The velocity

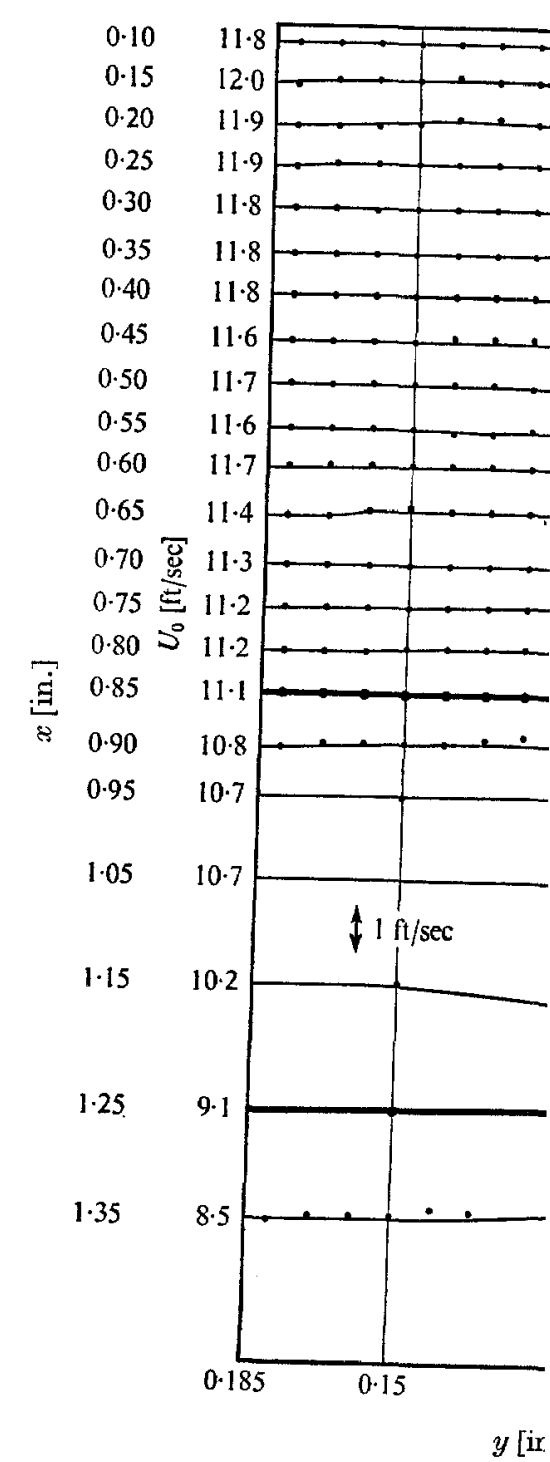

Figure 4. Transverse profiles o

defect $U_{0}-U$, where $U_{0}$ is the mean veloci with downstream distance $x$ for all crose the centre of the calm region. In other wo in the $x$ direction, $\partial\left(U_{0}-U\right) / \partial x$ is negative. positive values of this gradient (Schaefer

Figure 5 shows the mean velocity plot three cross-wake locations. The upper cur wake. The broken curve represents the the stagnation streamline in front of a circul fluid. The lower curve represents the veloc 
On the phenomenon of vortex street breakdown

511 nnel

rge tunnel

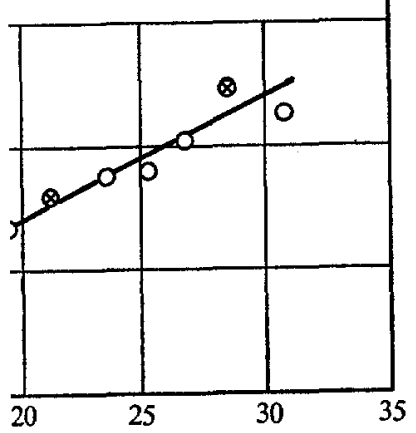

vs. primary wake frequency.

de in the large wind tunnel, thus the te shedding cylinder was $0.029 \mathrm{in}$. in ylinders was $1 \cdot 8 \mathrm{in}$. The free-stream sney $700 \mathrm{~Hz}$, and the air temperature

d r.m.s. fluctuations of the velocity, ymmetry of the large cylinder using isurements were made every $0.001 \mathrm{in}$. 05 in. in the downstream $(x)$ direction very $0 \cdot 1 \mathrm{in}$. in the calm and secondary yymmetrical, therefore, the measure-

zgainst $y$ for each downstream station. disappearance of the primary vortex urements. The second heavy curve at scondary vortex street. These profiles e the wake to a minimum on the wake ;he wake is symmetrical. The velocity

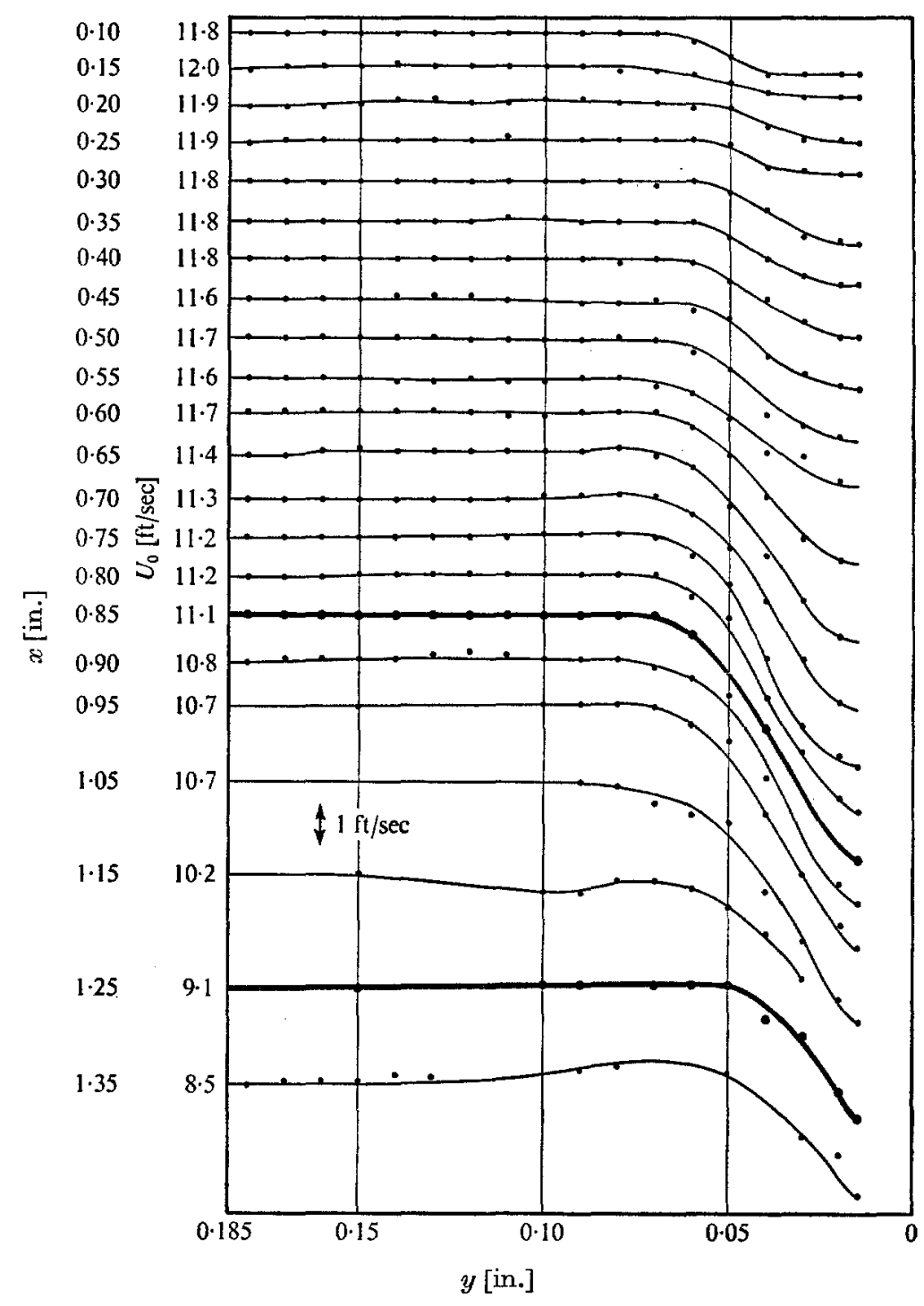

Figure 4. Transverse profiles of mean velocity in the wake.

defect $U_{0}-U$, where $U_{0}$ is the mean velooity outside the wake, is seen to increase with downstream distance $x$ for all cross-wake locations up to, approximately, the centre of the calm region. In other words, the mean deficit velocity gradient in the $x$ direction, $\partial\left(U_{0}-U\right) / \partial x$ is negative. Vortex streets in uniform flow exhibit positive values of this gradient (Schaefer \& Eskinazi 1959).

Figure 5 shows the mean velocity plotted against downstream distance for three cross-wake locations. The upper curve represents the velocity outside the wake. The broken curve represents the theoretical velocity distribution along the stagnation streamline in front of a circular cylinder in an unbounded inviscid fluid. The lower curve represents the velocity along the wake centreline, $U_{c}$. The 
velocity defect is very apparent here. The remaining curve shows similar variation at an intermediate position. A recovery in deficit velocity can be seen after the calm region. The foremost part of the large cylinder lies at $x=1.8 \mathrm{in}$.

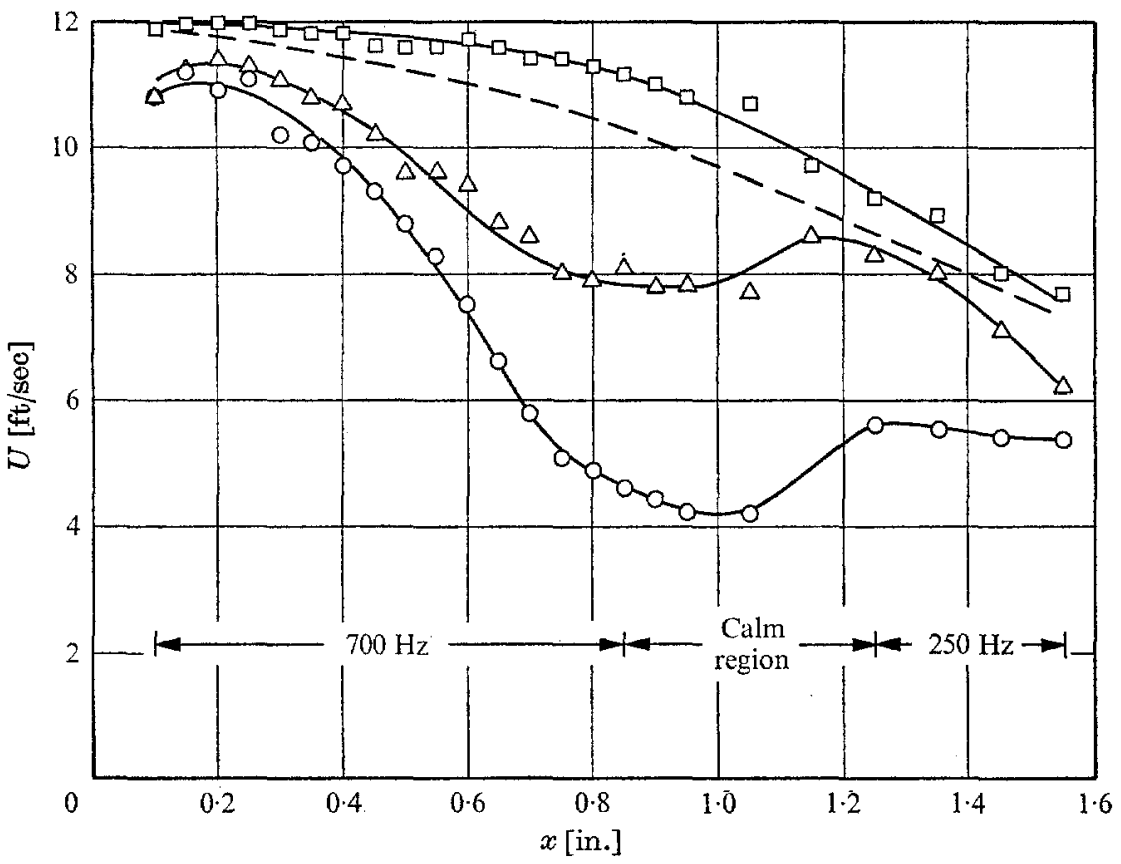

FIGURE 5. Longitudinal profiles of mean velocity in the wake. $\bigcirc, y=0$; $\triangle, y=0.25 ; \square, y=0.075 ;-\cdots$, , potential flow.

Figure 6 shows $\left(\overline{u^{2}}\right)^{\frac{1}{2}}$ plotted against $y$ for sequential downstream locations The slope of these curves is zero at the centreline, as it was for the mean velocity curves, because of the wake symmetry. The value is a minimum at the centreline increasing at first with $y$ until a maximum is achieved whereupon it begins to fall steadily to the value prevailing outside the wake. The maximum value as well as the area enclosed by these curves is seen to fall steadily with distance downstream until the formation of the new street at $x=1 \cdot 25 \mathrm{in}$. where $\left(\overline{u^{2}}\right)^{\frac{1}{2}}$ increases sharply. In this secondary street region there is at first a maximum on the centreline. Further downstream a minimum forms on the centreline with the maximum moving off-centre as in the primary street region.

The centreline minimum and off-centre maximum are characteristic (Schaefer \& Eskinazi 1959, Kovasznay 1949, Roshko 1953) of vortex streets in uniform flow as is the decay in the maximum value and area enclosed by the curves. The mean vorticity in the wake also is strongly affected. Although not directly measured we can obtain a fairly good estimate for it from our velocity measurements. The mean vorticity is given by

$$
\bar{\omega}=\partial \bar{v} / \partial x-\partial \bar{u} / \partial y
$$

where $\bar{u}$ and $\bar{v}$ are the mean velocities in the $x$ and $y$ directions respectively. It is easy to demonstrate from our measurements that $|\partial \vec{v} / \partial x| \ll|\partial \bar{u} / \partial y|$ in the wake.
Typically $|\bar{v} / \bar{u}| \simeq 0 \cdot 03, \mid(\partial \bar{v} / \partial x) /(\partial \bar{\imath}$ that

Figure 7 shows $-\bar{\omega}$ computed il for the same downstream locations the area under the curves is seen to beginning of the calm region wher diminishes in the secondary street net r.m.s. velocity falls in the prin observed in figure 5 are associated

\section{Geometrical structure of the v}

The curvature, or longitudinal sl by correlating the hot-wire signal $v$ located $1 \mathrm{in}$. above the stagnation hot-wire probe was moved downstrt when the signals were in phase, a $\mathrm{m}$ $\pm 90^{\circ}$ phase angle. Thus the zero cor the vortex positions.

Figure 8 shows the downstream $y=0.04 \mathrm{in}$. at various distances fror connecting these points represent $\mathrm{v}$.

It is evident that any given vort cylinder but rather begins somewh, off' the cylinder towards the plane o the large cylinder and get closer $\mathbf{t}$ secondary street region, correlation metry of the large cylinder $(z=0)$. as they travel downstream.

In the primary vortex street regio: location, $x$, of the vortices at a given between successive locations was ta: downstream location given by the me values are plotted in figure 9. Vali subsequent calculations were taker decrease in downstream spacing is and the value just as the primary $v$ noted, this behaviour deviates cons: uniform flow which exhibits a relati (Schaefer \& Eskinazi 1959).

Early measurements of the dista spacing) were made using photogra street. Any point about which the flu centre and spacing measured from 


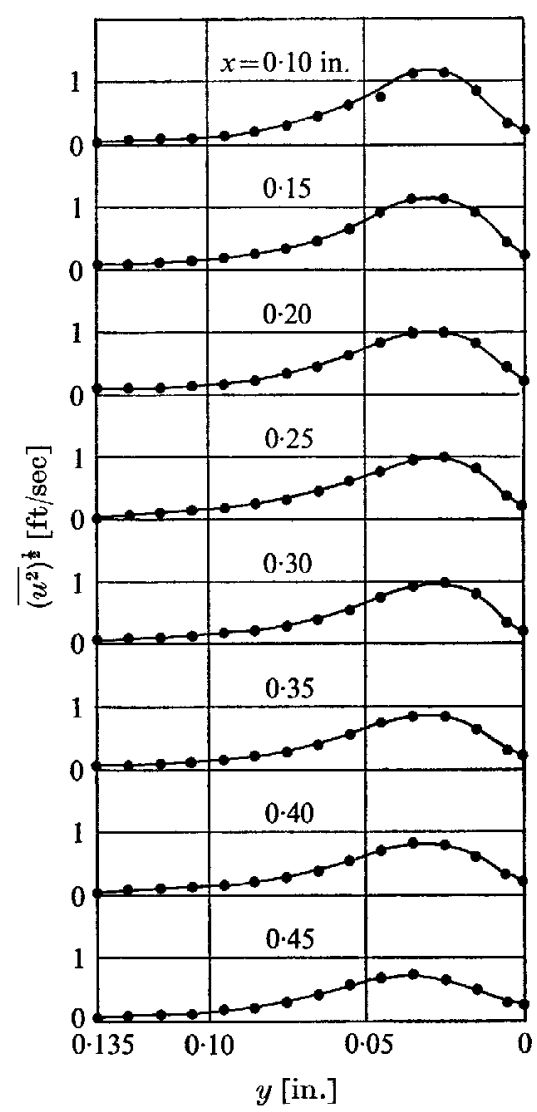

(a)

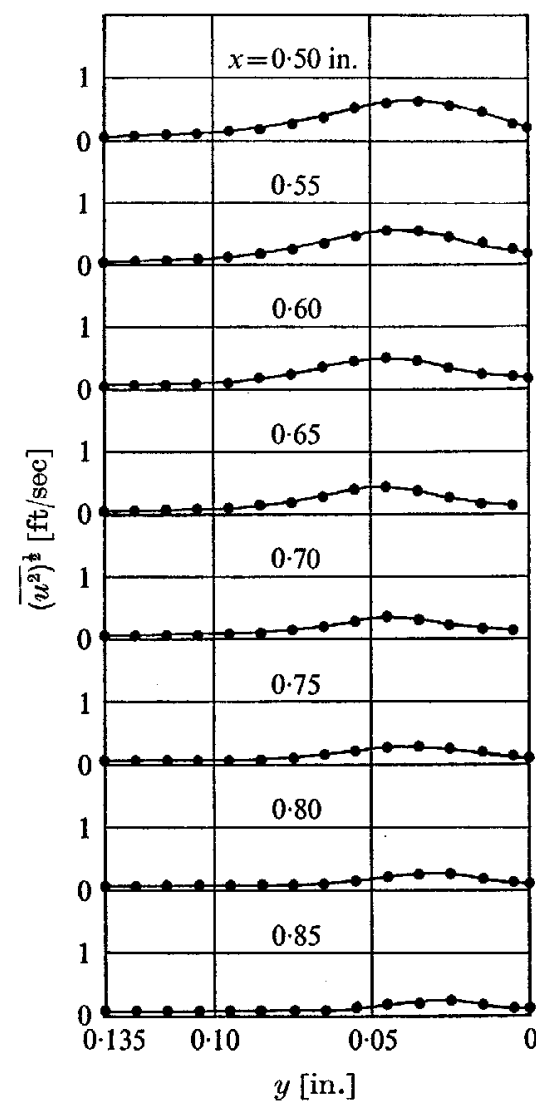

(b)

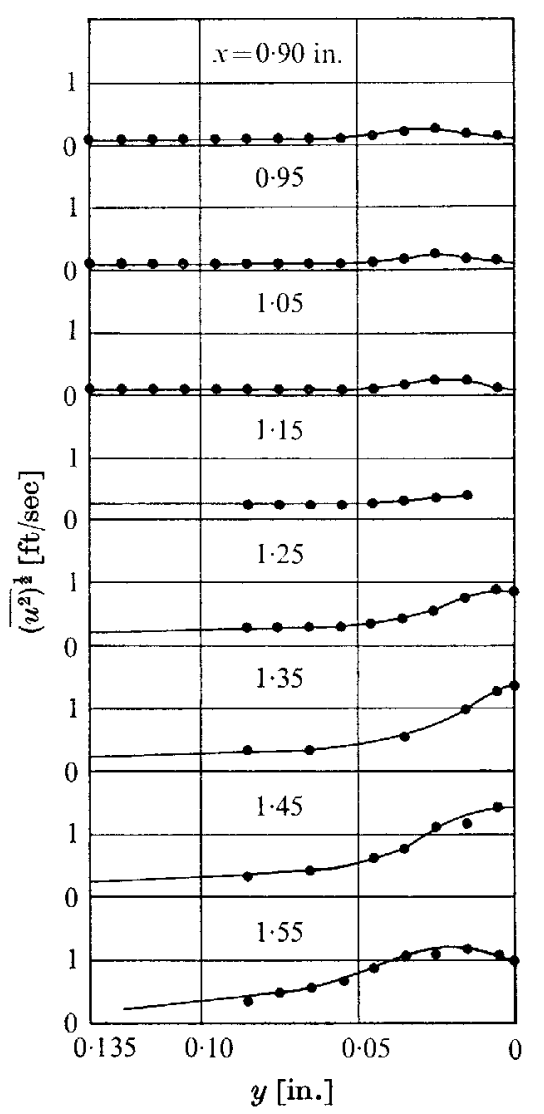

(c)

FtaURe 6. Profiles of r.m.s. velocity fluctuations
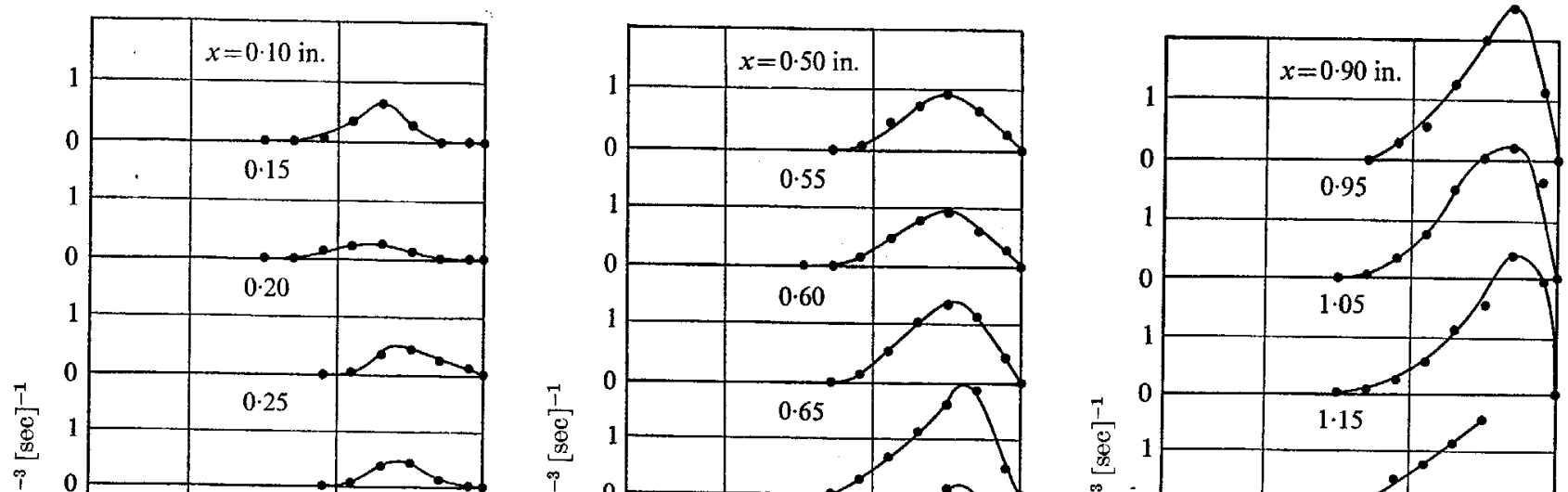


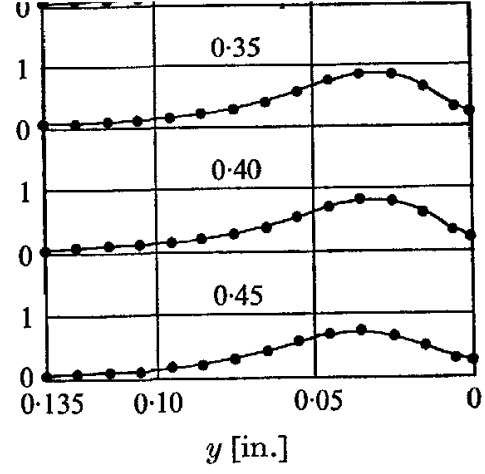

(a)

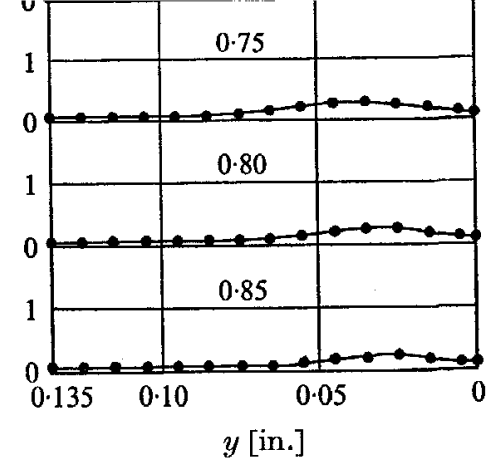

(b)

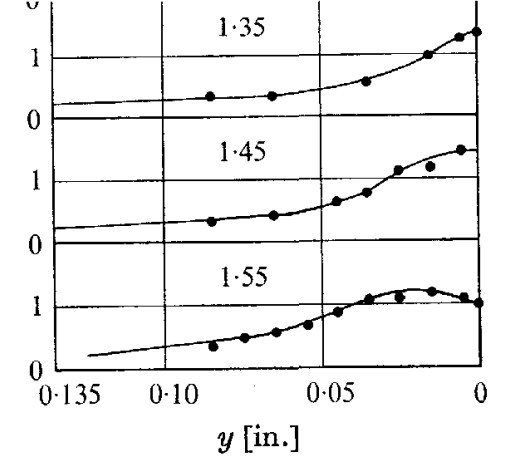

(c)

Frgure 6. Profiles of r.m.s. velocity fluctuations.

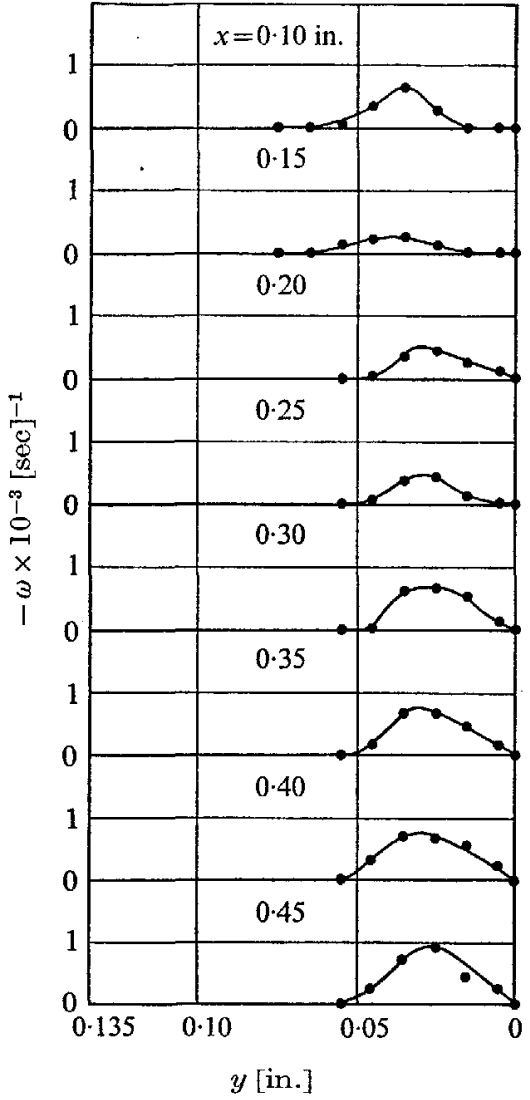

(a)

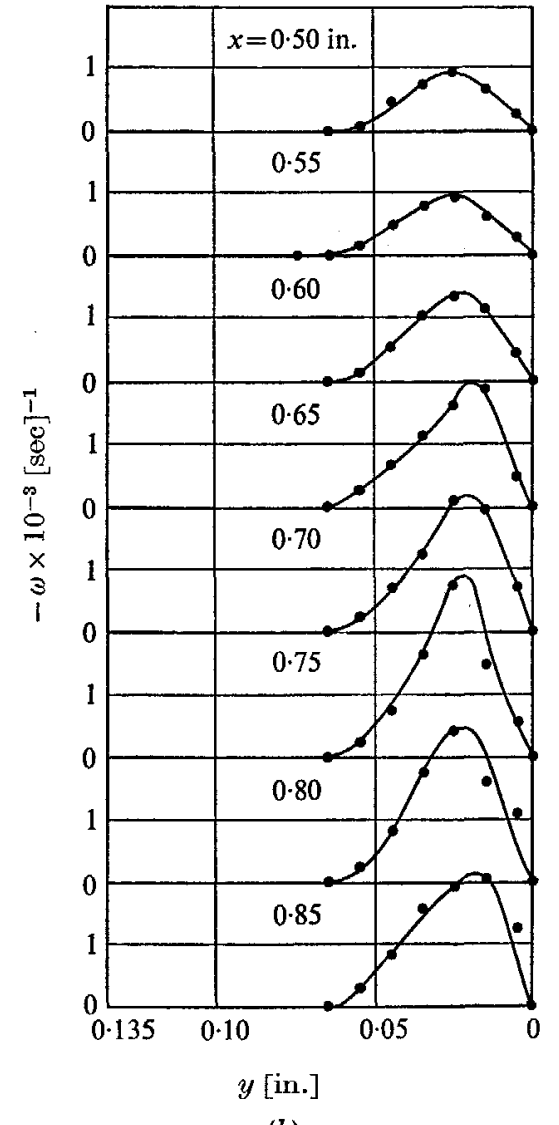

(b)

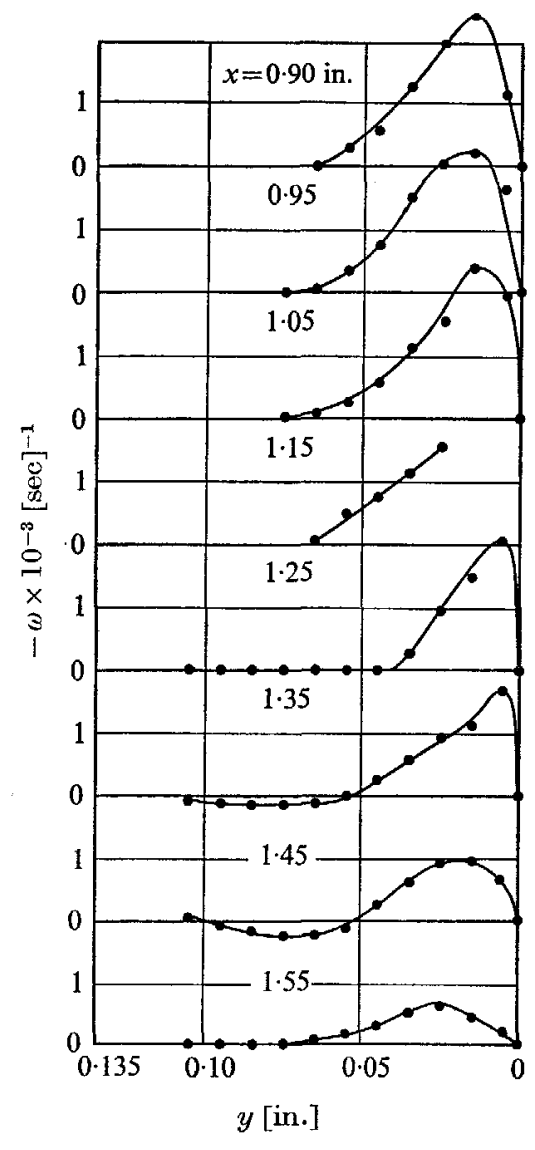

(c)

Figure 7. Mean vorticity.

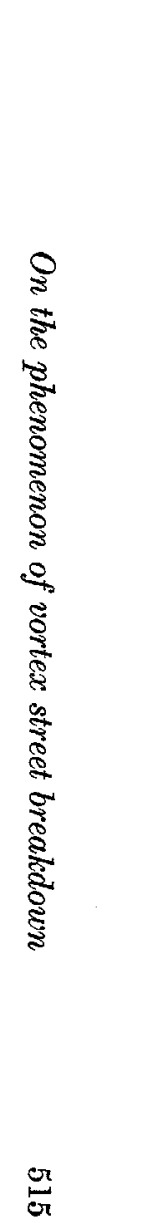


points differed from the point of maximum vorticity or actual vortex centre because of the diffusion of vorticity. He showed how to correct for this error by estimating the age, $\tau$, of the vortices and assuming them to diffuse in the HamelOseen fashion, (cf. equation (3)).

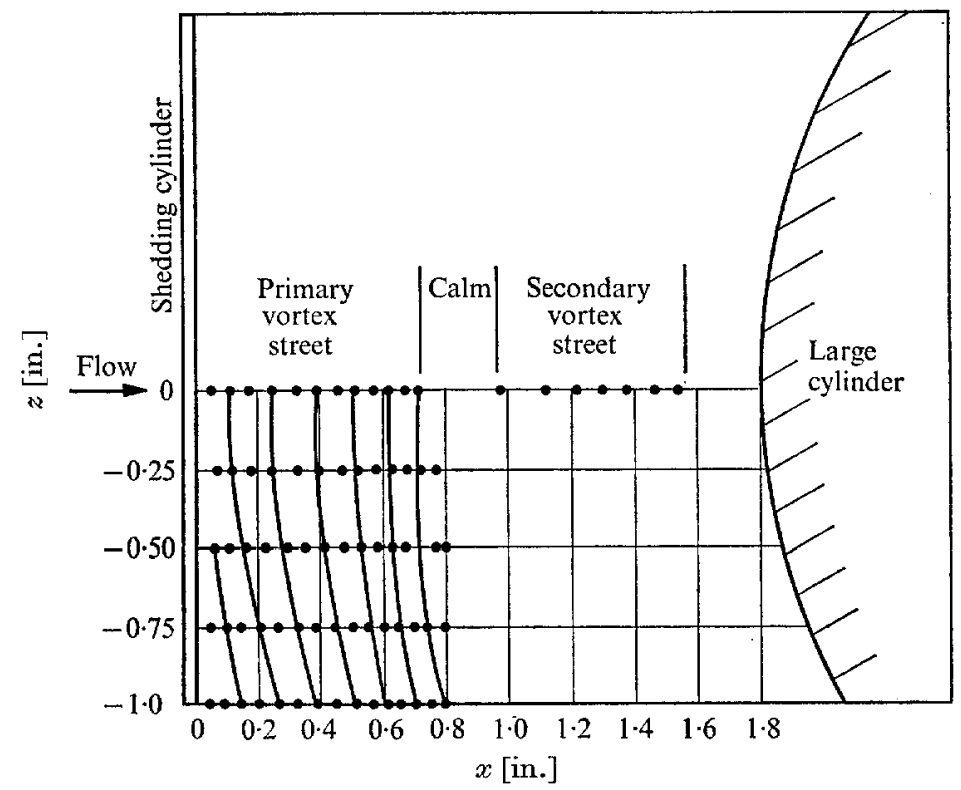

Figure 8. Correlation zeros.

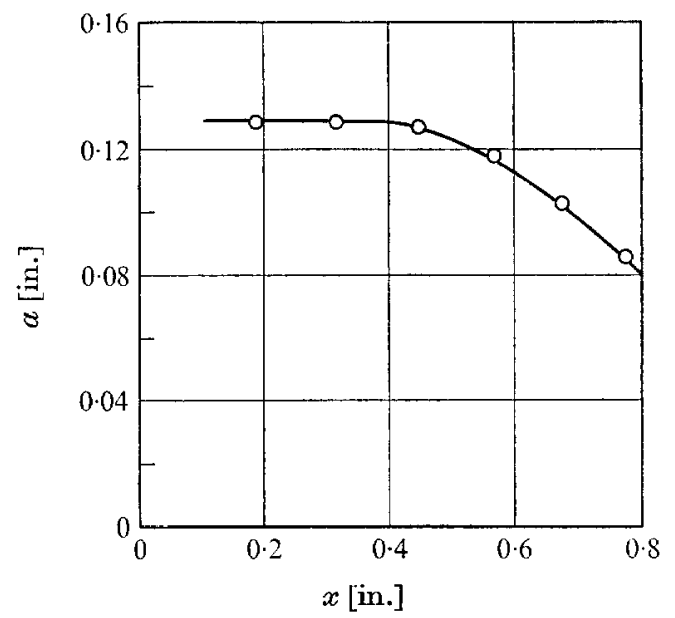

Figure 9. Downstream spacing.

Schaefer \& Eskinazi adapted essentially the same method for use with hotwire anemometry. They assumed the vortices to be of the Hamel-Oseen type whence the maximum peripheral velocity is at a distance of $r_{*}=(5 \cdot 04 v \tau)^{\frac{1}{2}}$ from the vortex centre. Using the additional assumption that the maximum in the cross-wake plot of r.m.s. velocity coincides with the outside maximum peripheral velocity, they calculated the laters where $h_{*}$ is the distance between th Eskinazi obtained good theoreti justifying their assumptions, the vor not circular and therefore the applic able.

A reasonable assumption here $i$ vortex centres coincides with the approximation $\bar{\omega} \approx-\partial \bar{u} / \partial y$, this cer wake mean velocity plots. An exa indeed show that for their vortex s maximum slopes of their mean veloc values of $h$.

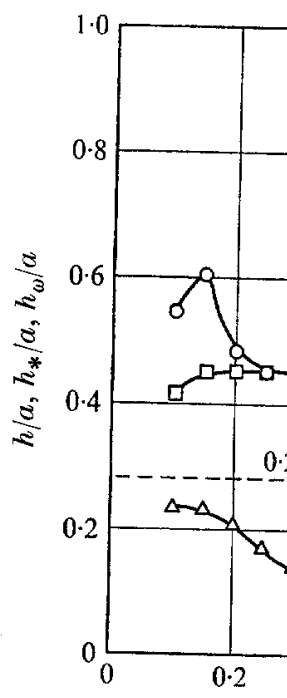

Figure 1

Figure 10 is a comparison betwee and $h$ as functions of $x$, all normalize For comparison, von Kármán's valu $\tau=\tau(x)$ obtained from the measurem to traverse a vortex spacing given by

Whereas the data of Schaefer \& $\mathbf{E}$ figure 10 reveals that, for the preser across-wake spacing ratio based on substantially greater than that calct maxima and vortex age, $h / a$, indicat vorticity.

The phase of the hot-wire signal re from photographs of oscilloscope tra 


\section{F. Karlsson}

vorticity or actual vortex centre how to correct for this error by ing them to diffuse in the Hamel-

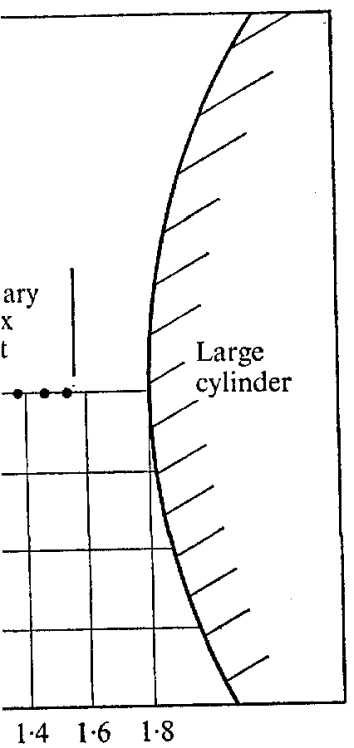

in zeros.

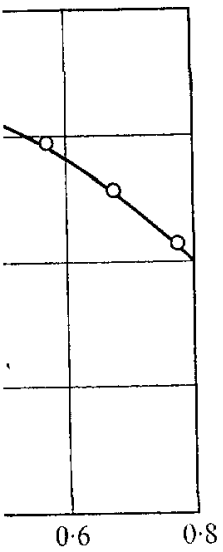

m spacing.

ihe same method for use with hotes to be of the Hamel-Oseen type at a distance of $r_{*}=(5 \cdot 04 v \tau)^{\frac{1}{2}}$ from imption that the maximum in the th the outside maximum peripheral
On the phenomenon of vortex street breakdown

velocity, they calculated the lateral spacing of the vortex rows as $h=h_{*}-2 r_{*}$ where $h_{*}$ is the distance between the r.m.s. velocity peaks. Although Schaefer \& Eskinazi obtained good theoretical and experimental agreement, thereby justifying their assumptions, the vortices in the present case are (as will be shown) not circular and therefore the applicability here of the above method is questionable.

A reasonable assumption here is that the across-wake location, $h_{\omega}$, of the vortex centres coincides with the maximum in the mean vorticity. Using the approximation $\bar{\omega} \approx-\partial \bar{u} / \partial y$, this centre is also the maximum slope of the acrosswake mean velocity plots. An examination of Schaefer \& Eskinazi's data did indeed show that for their vortex street the across-wake distance between the maximum slopes of their mean velocity plots, $h_{\omega}$, was identical to their computed values of $h$.

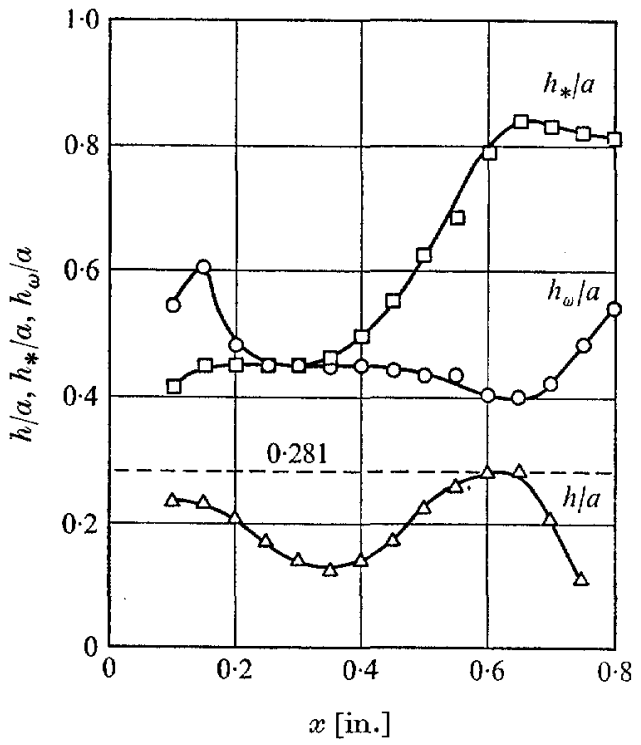

Figure 10. Spacing ratios.

Figure 10 is a comparison between the different cross-wake spacings $h_{*}, h_{\omega}$, and $h$ as functions of $x$, all normalized by the local downstream spacing ratio $a$. For comparison, von Kármán's value of 0.281 is also shown. Figure 11 shows $\tau=\tau(x)$ obtained from the measurements by noting that a vortex took $1 / 700 \mathrm{sec}$ to traverse a vortex spacing given by figure 9 .

Whereas the data of Schaefer \& Eskinazi show $h_{\omega} / a$ and $h / a$ to be coincident, figure 10 reveals that, for the present case, there is a marked difference. The across-wake spacing ratio based on mean vorticity maxima, $h_{\omega} / a$, is always substantially greater than that calculated from the spacing of r.m.s. velocity maxima and vortex age, $h / a$, indicating a reduction in the lateral diffusion of vorticity.

The phase of the hot-wire signal relative to the reference signal was measured from photographs of oscilloscope traces representing the velocity fluctuations 
and the reference signal. These measurements were made at several across-wake locations for various downstream locations. Figure 12 shows the phase angle plotted versus $y$ for fixed values of $x$, the downstream stations. The inside of a vortex, i.e. the part nearest $y=0$, is seen to lag the outer part for all but one curve. This lag becomes especially pronounced, almost a full cycle, just prior to the calm region. The missing points of the last two frames correspond to weak signals whose phase could not be reliably determined.

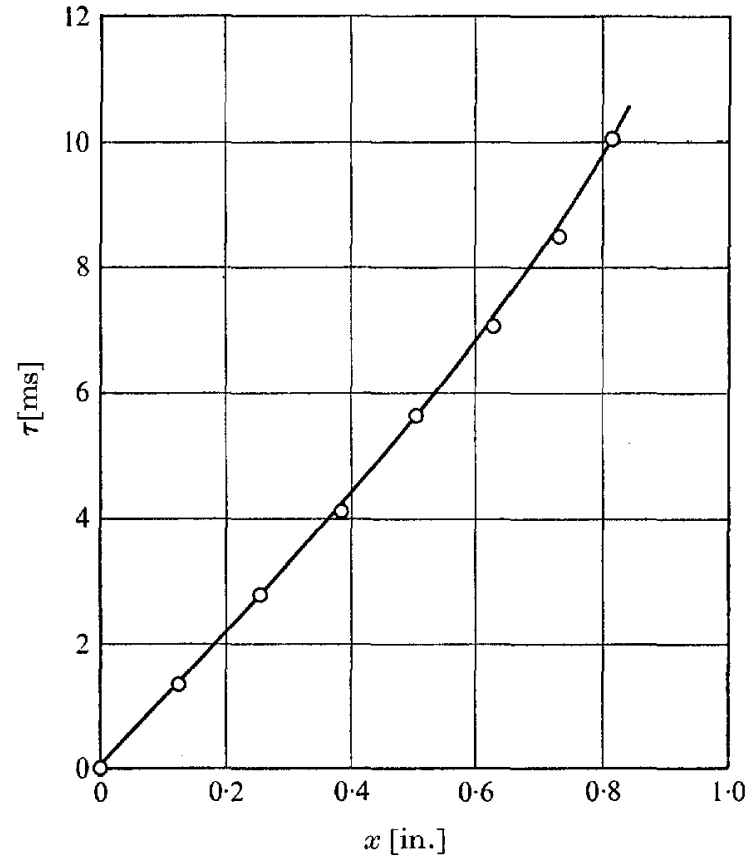

Figure 11. Vortex age.

Figure 13 shows wave analyses of the hot-wire signal at various downstream positions along the wake centreline. The band pass of the wave analyzer was $\frac{1}{3}$ octave and the vertical axis represents $\left(\bar{u}^{2}\right)^{\frac{1}{2}}$. The data was taken in the small wind tunnel using a cylinder separation of $1.2 \mathrm{in}$. and a primary wake frequency of $670 \mathrm{~Hz}$. The curve at $x=0 \cdot 24 \mathrm{in}$. shows a peak at the primary frequency and lesser peaks at the first and second harmonics. The curve at $x=0.4$ shows peaks at the fundamental and first harmonic only. These locations were in the primary vortex street region. At $x=0.6$ and $0.7 \mathrm{in}$. an entirely different spectrum is present; this is the secondary vortex street region. The fundamental of $240 \mathrm{~Hz}$ presents the largest peak and first and second harmonics are visible. At any station, if the probe was moved away from the centreline, the harmonics would quickly disappear and the fundamental gradually die in amplitude.

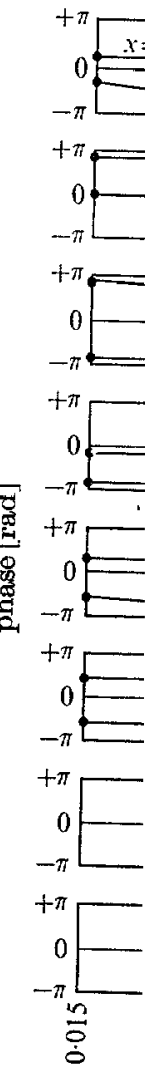

FIgUre 12. Relative phas

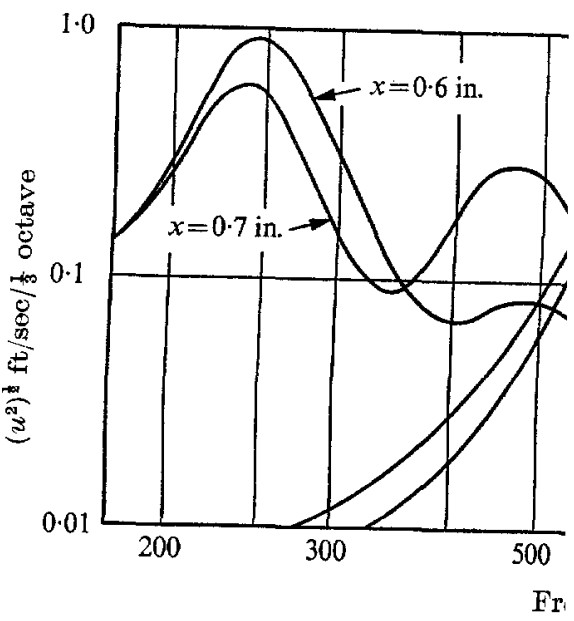

Figure 13. Wave analy 


\section{Karlsson}

made at several across-wake ure 12 shows the phase angle ream stations. The inside of a the outer part for all but one almost a full cycle, just prior wo frames correspond to weak ned.

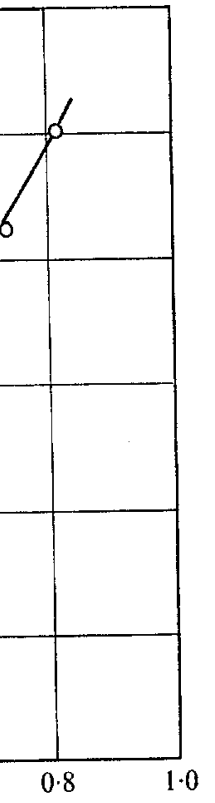

e signal at various downstream pass of the wave analyzer was he data was taken in the small and a primary wake frequency $\mathrm{k}$ at the primary frequency and he curve at $x=0 \cdot 4$ shows peaks se locations were in the primary entirely different spectrum is on. The fundamental of $240 \mathrm{~Hz}$ harmonics are visible. At any entreline, the harmonics would ly die in amplitude.
On the phenomenon of vortex street breakdown
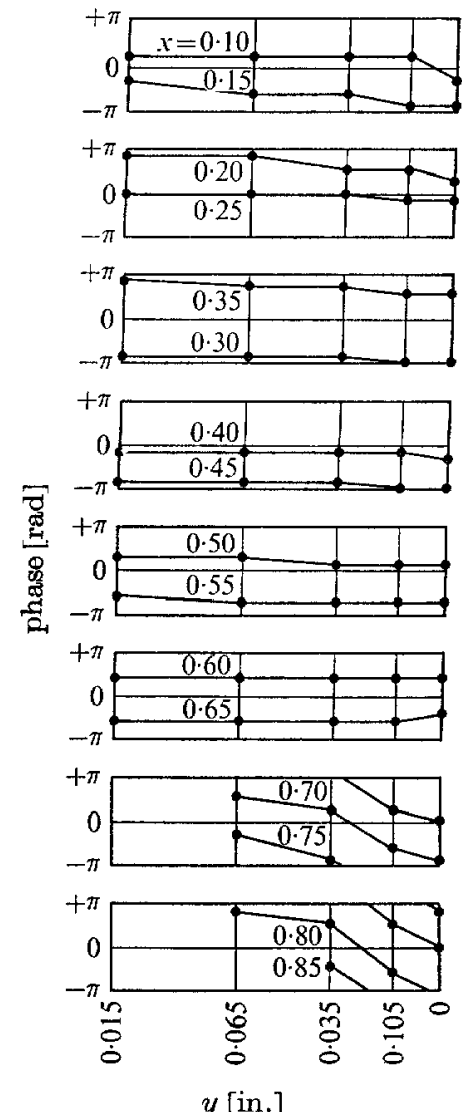

Figure 12. Relative phase angle of velocity fluctuations.

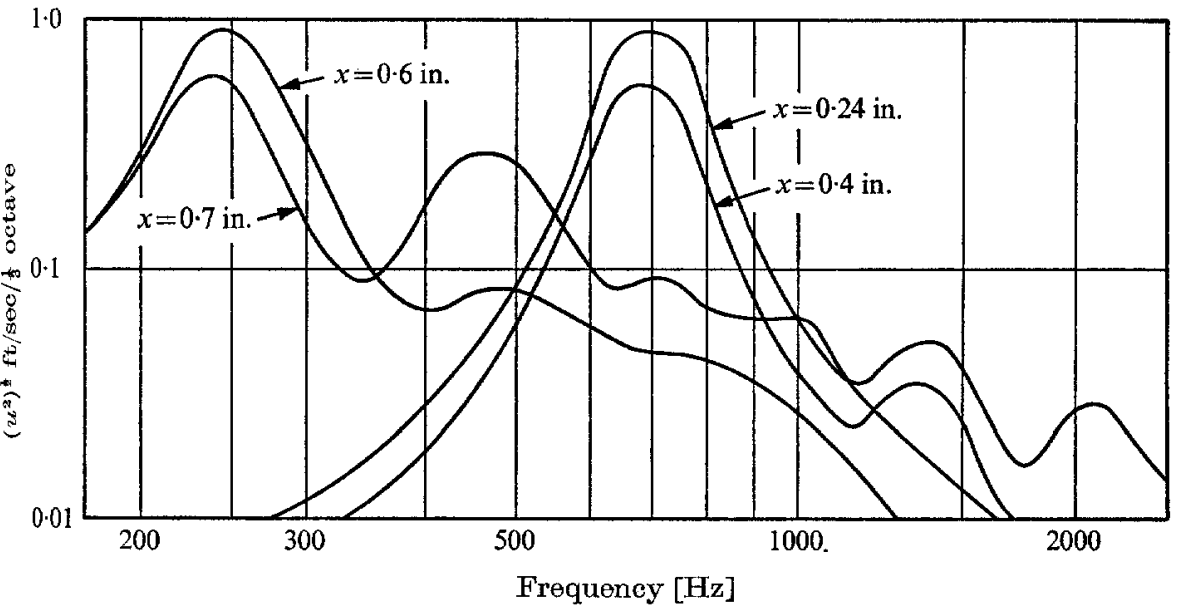

FIGURE 13. Wave analysis along the wake centreline. 


\section{Other relevant experiments}

Two experimental investigations into vortex street breakdown by Taneda (1959) and Zdravkovich (1968) are of particular interest in connexion with the present work.

Using a glass-sided towing tank Taneda was able to observe the wakes behind towed cylinders and plates for very long distances via the aluminium dust, side slit lighting technique. His improved experimental techniques allowed much more extended observations of the vortex street development than had been possible in earlier wind-tunnel investigations where typically the free-stream velocity fluctuations due to background turbulence were comparable in magnitude to fluctuations in the vortex street at 30 diameters downstream from the shedding cylinder, effectively terminating the study of further vortex street development.

For Reynolds numbers less than 150, Taneda found the primary vortex street lasted for about 50 cylinder diameters before breaking down. The secondary vortex street formed slowly, reaching full development about $10^{3}$ diameters downstream. He found the secondary vortex street wavelength to be between 1.8 and 3.3 times the primary wavelength. For Reynolds numbers greater than 150 he found that the vortex street breaks down to turbulence and then forms a secondary vortex street with about 10 times larger wavelength than the primary one. In a more recent investigation Taneda (1965) has found that the turbulent wake régime behind a flat plate also shows a strong tendency to become unstable and form a vortex street.

Taneda also found that the secondary vortex street either broke down forming a third vortex street or died out through viscous diffusion.

Zdravkovich qualitatively studied the wake of a group of three cylinders using smoke to make the wake visible in a vertical wind tunnel. By careful positioning of the three cylinders he was able to generate single vortex streets with characteristically large spacing ratios (e.g. $0 \cdot 67$ for one experiment). From his photographs it can be seen that the vortices of this vortex street continually deform; the outside of a vortex appears to advance and the inside slows down relative to the 'vortex centre'. The vortices thus become somewhat oval in shape finally aligning themselves with the mean flow direction and become in distinguishable. Sinusoidal oscillations are then seen to arise and develop into

$x$ in phase (in.)
$0 \cdot 123$
$0 \cdot 252$
$0 \cdot 381$
0.509
$0 \cdot 627$
$0 \cdot 730$
$0 \cdot 816$

$\begin{array}{cc}a \text { spacing (in.) } & x \text { location of } a \text { (in.) } \\ 0.129 & 0.118 \\ 0 \cdot 129 & 0.316 \\ 0 \cdot 128 & 0.445 \\ 0 \cdot 118 & 0.568 \\ 0 \cdot 103 & 0.678 \\ 0.086 & 0.773\end{array}$

TABLE I a new vortex street of larger wav is usually about 2 to 1.

The relatively large spacing ra vortex street behind three cylin apparently governs in some way circular shape to one conducive of the secondary vortex street.

\section{Discussion}

There are essentially three diff the vortex street breakdown in $t$ the vortex street behind a cylin filaments curve about the large cy of the mean flow around the larg Thirdly, the vortices grow closer $t$ travel downstream because the $m$ Neither Taneda's nor Zdravkovic or stretching of the vortex filam behaviour similar to that observ experiment was, however, the larg forced to have because of the me experiments, bending and stretch primary causes of vortex street $b$ breakdown process sought, which strongly on the spacing ratio of th

Taking the same point of view and letting the flow in the vortex position, we see that any one vorte all the others. It is thus appropria of one selected vortex by all the ot

Shortly after formation close to vortex street is a highly concentra called the vortex centre. If, initia infinitesimal filament of circulatio bounded, the ensuing diffusion of $\mathrm{v}$

$$
\omega=\frac{1}{4 \pi}
$$

where $r^{2}=x^{2}+y^{2}$ and the co-ordin In this solution the vorticity diffu creating a 'diffuse' vortex or patch

At distances $r \gg(\nu \tau)^{\frac{1}{2}}$ from the ce to a potential vortex. In fact, a c radius of maximum induced peripl 
a new vortex street of larger wavelength than the original. The wavelength ratio is usually about 2 to 1 .

The relatively large spacing ratio is the only obvious difference between the vortex street behind three cylinders and that behind a single cylinder. This apparently governs in some way the deformation of each vortex from its usually circular shape to one conducive to the conditions which precede the formation of the secondary vortex street.

\section{Discussion}

There are essentially three differences between the flow field associated with the vortex street breakdown in the present case and the flow field surrounding the vortex street behind a cylinder in an unbounded fluid. First, the vortex filaments curve about the large cylinder as shown in figure 8 . Secondly, by virtue of the mean flow around the large cylinder, the vortex filaments are stretched. Thirdly, the vortices grow closer together (in the downstream direction) as they travel downstream because the mean flow is slowing as shown in figures 8 and 9 . Neither Taneda's nor Zdravkovich's experiments apparently involved bending or stretching of the vortex filaments on any appreciable scale yet they show behaviour similar to that observed here. The unique feature of the latter's experiment was, however, the large spacing ratio the primary vortex street was forced to have because of the method of its generation. On the basis of these experiments, bending and stretching will be tentatively ruled out as possible primary causes of vortex street breakdown, and a mechanism to describe the breakdown process sought, which is two-dimensional in nature and depends strongly on the spacing ratio of the primary vortex street.

Taking the same point of view as von Kármán (and, of course, others later) and letting the flow in the vortex street be given by the vortices in type and position, we see that any one vortex can be deformed by the combined action of all the others. It is thus appropriate to examine, in some detail, the convection of one selected vortex by all the other vortices.

Shortly after formation close to the shedding cylinder a vortex in the primary vortex street is a highly concentrated distribution of vorticity about some point called the vortex centre. If, initially, the vorticity is all concentrated into an infinitesimal filament of circulation $\Gamma$ (potential vortex), and the field is unbounded, the ensuing diffusion of vorticity is given by the Hamel-Oseen solution

$$
\omega=\frac{\Gamma}{4 \pi \nu \tau} \exp \left(-\frac{r^{2}}{4 \nu \tau}\right)
$$

$0 \cdot 118$

$0 \cdot 316$

$0 \cdot 445$

0.568

0.678

$0 \cdot 773$

where $r^{2}=x^{2}+y^{2}$ and the co-ordinate system is fixed in the centre of the vortex. In this solution the vorticity diffuses radially outward from the vortex centre creating a 'diffuse' vortex or patch of vorticity.

At distances $r \gg(\nu \tau)^{\frac{1}{2}}$ from the centre of a Hamel-Oseen vortex, it is identical to a potential vortex. In fact, a circle with the radius $r=r_{*}=(5 \cdot 04 \nu \tau)^{\frac{1}{2}}$, the radius of maximum induced peripheral velocity, includes approximately $70 \%$ 
of the vorticity associated with the vortex. Providing none of the vortices 'overlap', that is $\left(\frac{1}{2} a\right)^{2}+h^{2}>4 r_{*}^{2}$ and $a>2 r_{*}$ where $h$ is used for the distance between rows since there is no ambiguity here, we may say that to a good approximation all the vortices look like potential vortices to the one under consideration. Further assuming a constant downstream spacing, $a$, and across-wake spacing, $h$, it can be shown (Durgin 1970) that the initially circular cross-section of the

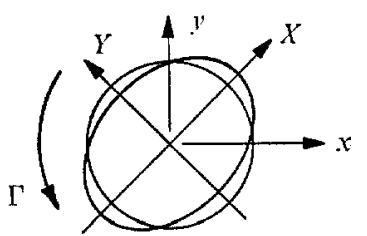

(a) $\alpha>0$

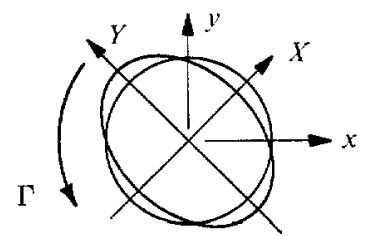

(b) $\alpha<0$
FIGURE 14. Modes of vortex straining.

vortex region is in general convected into an elliptic shape. More precisely, a circle about $(0,0)$ given by

$$
C(x, y, t=0)=(x / R)^{2}+(y / R)^{2}=1,
$$

is, to second order of approximation, deformed into

$$
C(x, y, t)=(x / R)^{2} \cosh (2 \alpha t)-2\left(x y / R^{2}\right) \sinh (2 \alpha t)+(y / R)^{2} \cosh (2 \alpha t)=1 .
$$

This is the equation of an ellipse which upon rotation of the co-ordinate system by $45^{\circ}$ to the principal axes becomes

$$
C(X, Y, t)=\left(X \mid R e^{\alpha t}\right)^{2}+\left(Y \mid R e^{-\alpha t}\right)^{2}=1 .
$$

If $\alpha=0$ the curve $C$ remains circular. If $\alpha>0$ the original circle deforms with time as shown in figure $14(a)$. The major axis of the ellipse lies on the $X$ axis while the minor lies on the $Y$ axis. If $\alpha<0$ the reverse situation is true as shown in figure $14(b)$ with the major axis of the ellipse on the $Y$ axis and the minor axis on the $X$ axis.

The parameter $\alpha$ is a function of the vortex spacing ratio which has been plotted in figure 15 in terms of $2 \pi a^{2} \alpha / \Gamma v s$. $h / a$. It can be seen that $\alpha>0$ if $h / a<0.366$ and $\alpha<0$ if $h / a>0 \cdot 366$. Thus the fluid near the centre of any vortex can be strained into two possible modes depending on the values of the spacing ratio.

As soon as a vortex deviates from its idealized circular shape its shape can be further changed by its own induced velocities. For the purpose of finding the self-induced motion of the elliptically deformed vortex it is convenient to assume that all the vorticity associated with the vortex is initially uniformly distributed within a specified radius, say $r_{*}$. This uniform vorticity is given by

$$
\omega=\Gamma / \pi r_{*}^{2}
$$

and the ellipse bounding the en replacing $R$. The principal axes o

$$
c=\imath
$$

The area of the ellipse, $\pi c b$, is co uniform distribution of vorticity

A uniform elliptical vorticity $d$ velocity (Lamb 1945, p. 232)

Thus the elliptical vorticity dist in the same sense as its circulat deformation increases.

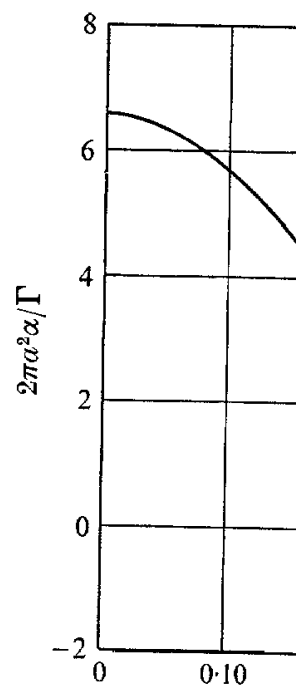

FiguRe 15. Th

As soon as a vorticity distributior the other vortices induce, and conse the problem of finding the motion and the foregoing analysis is not $\mathrm{s}$ clear, however, that the same proce qualitatively some insight may be discrete.

\section{Comparison with experiment}

The phenomenon observed in the than 0.366 and thus in the $\alpha<0$ ré the part of a vortex outside of the $v$ stream direction. This mode is in ag? variations (figure 12). 
At $x=0.65$ (just preceding serious phase shifts) the downstream spacing $a=0.107$ in. and the spacing ratio $h_{\omega} / a=0.4$ which will be taken to be $h / a$ for the purpose of this comparison. The circulation around any vortex is given by

$$
\Gamma=\int_{A} \omega d a
$$

providing the vortices do not overlap and the integration is only carried out over the vorticity belonging to the vortex under consideration. Neglecting diffusion in the downstream direction the mean vorticity flux per unit depth through a plane, $x=$ const. from $y=-\frac{1}{2} h$ to $\infty$ (here $-\frac{1}{2} h$ is the wake centreline) is then $f \Gamma$ where $f$ is the frequency at which vortices pass a fixed $x$. But the mean vorticity flux may also be written

$$
f \Gamma=\int_{-\frac{1}{2} \hbar}^{\infty} \overline{u \omega} d y .
$$

The integrand may be expanded to read

$$
\overline{u \omega}=\bar{u} \frac{\partial \bar{v}}{\partial x}-\bar{u} \frac{\partial \bar{u}}{\partial y}+\overline{u^{\prime} \frac{\partial v^{\prime}}{\partial x}}-\overline{u^{\prime} \frac{\partial u^{\prime}}{\partial y}} .
$$

Neglecting $\bar{u}(\partial \bar{v} / \partial x)$ and $\overline{u^{\prime}\left(\partial v^{\prime} / \partial x\right)}$ in comparison with the other terms the circulation becomes

$$
\Gamma=\frac{-1}{2 f}\left[\bar{u}^{2}+\overline{u^{\prime 2}}\right]_{-\frac{1}{2} h}^{\infty} .
$$

Substituting the appropriate values for $x=0.65 \mathrm{in}$. from figures 4 and 6 and $f=700 \mathrm{~Hz}$ the circulation around a vortex at $x=0.65 \mathrm{in}$. is $\Gamma=9.9 \mathrm{in} .{ }^{2} / \mathrm{sec}$. From figure 11 the vortex age is found, whence $r_{*}=0.03 \mathrm{in}$. From figure 15 $2 \pi a^{2} \alpha / \Gamma=0.56$, when $\alpha=76$. Using $t=1 / 700 \mathrm{sec}$ in equation (7)

$$
c / r_{*}=1 \cdot 12, \quad b / r_{*}=0 \cdot 89
$$

in one cycle. From equation (8) the corresponding rotation rate becomes $n=861 \mathrm{rad} / \mathrm{sec}$ or in terms of rotation, $\theta$, the ellipse would rotate $\theta=70^{\circ}$ in one cycle. Evidently, this estimate is too large, because rotation of this magnitude does not occur in the experiment. Clearly, simple superposition of the two mechanisms at work here is not valid for that large deformations.

Limitation on the extent of rotation in the present model can be explained by noting that as a strained vortex rotates its extremes enter regions of large velocities induced by its neighbours of the same row which are undergoing similar processes. These velocities counteract the self-induced rotation of the deformed vortex. As the vortices rotate, they assume some alignment in the downstream direction whereupon the character of the convection has changed drastically. This alignment causes the vortices to become indistinguishable from one another and, in essence, a shear layer is formed in each row. These shear layers would correspond to what has previously been called the calm region.

\section{Stability of the calm region}

An examination of the mean veloc profiles, figure 7 , reveals that the ca vorticity of opposite signs. These pr formed behind a flat plate in a uni flow to sinusoidal perturbations in $x$ (1961). Although their analysis was interesting, nevertheless, for the pur results to the present case, overlooki Figure 16 shows the mean velocity a compared to their velocity distributi $\left(U_{0}-U_{c}\right) / U_{0}=0.609$ for the present ca

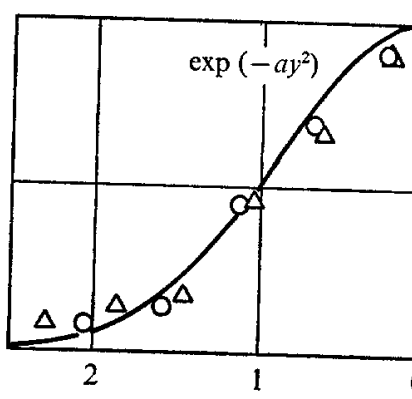

Frgure 16. Non-dimensional vel

$$
\bigcirc, x=0 \text {. }
$$

Computing the frequency of the $n$ Sato \& Kuriki, it is found that $\sigma=$ $x=0 \cdot 95$. These values are somewhat tally. It should be noted that Sato \& experimental values for Reynolds num? 500. The same Reynolds number for relatively low value of the Reynolds effects are not completely negligible. Un unknown, is the influence of the decel The analysis does, however, predict a $700 \mathrm{~Hz}$ primary wake frequency.

\section{Conclusions}

Throughout this study of vortex stre of view that a certain distribution of the wake. This idea was originated by of velocities induced by potential vorti down, we have found that it is necess 


\section{F. Karlsson}

shifts) the downstream spacing which will be taken to be $h / a$ for $n$ around any vortex is given by

ntegration is only carried out over onsideration. Neglecting diffusion ity flux per unit depth through a $\frac{1}{2} h$ is the wake centreline) is then ss a fixed $x$. But the mean vorticity

$l y$.

$$
\overline{\frac{v^{\prime}}{x}}-\overline{u^{\prime} \frac{\partial u^{\prime}}{\partial y}}
$$

In with the other terms the circula-

$\overline{2}]_{-\frac{1}{2} h}^{\infty}$.

0.65 in. from figures 4 and 6 and at $x=0.65$ in. is $\Gamma=9.9 \mathrm{in} .{ }^{2} / \mathrm{sec}$. tence $r_{*}=0.03 \mathrm{in}$. From figure 15 0 sec in equation (7)

$=0 \cdot 89$

esponding rotation rate becomes he ellipse would rotate $\theta=70^{\circ}$ in , because rotation of this magnitude simple superposition of the two $t$ large deformations.

le present model can be explained its extremes enter regions of large same row which are undergoing ct the self-induced rotation of the ey assume some alignment in the ter of the convection has changed is to become indistinguishable from $\mathrm{s}$ formed in each row. These shear isly been called the calm region.
On the phenomenon of vortex street breakdown

525

\section{Stability of the calm region}

An examination of the mean velocity profiles, figure 4 , and the mean vorticity profiles, figure 7 , reveals that the calm region consists of two adjacent layers of vorticity of opposite signs. These profiles are reminiscent of the laminar wake formed behind a flat plate in a uniform stream. The stability of this type of flow to sinusoidal perturbations in $x$ and $t$ has been studied by Sato \& Kuriki (1961). Although their analysis was developed for uniform external flow it is interesting, nevertheless, for the purpose of a rough comparison, to apply the results to the present case, overlooking for the moment this obvious difference. Figure 16 shows the mean velocity at $x=0.85$ and 0.95 in. for the present case compared to their velocity distribution. The agreement is reasonably good but $\left(U_{0}-U_{c}\right) / U_{0}=0.609$ for the present case as compared to 0.692 for Sato \& Kuriki.

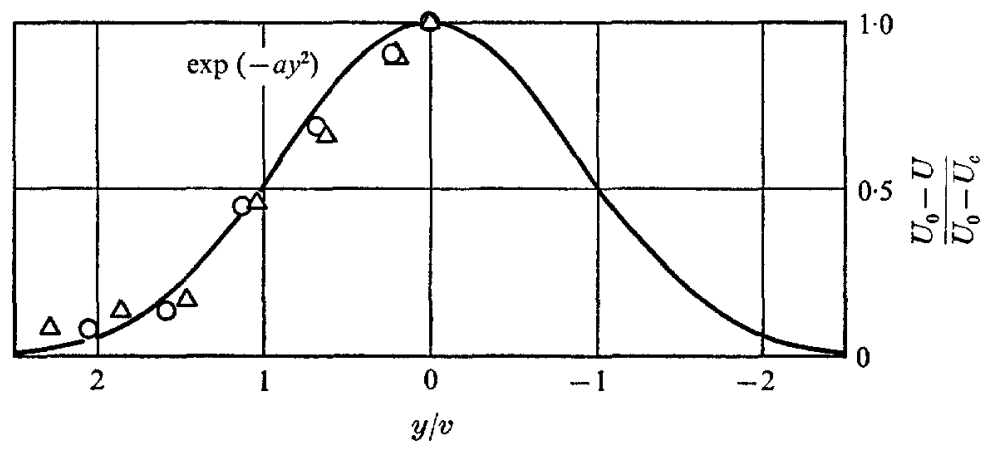

FrgURE 16. Non-dimensional velocity distribution in the calm region.

$$
\mathrm{O}, x=0 \cdot 85 ; \triangle, 0 \cdot 95 \text {. }
$$

Computing the frequency of the most amplified scale from the results of Sato \& Kuriki, it is found that $\sigma=465 \mathrm{~Hz}$ for $x=0.85$ and $\sigma=426 \mathrm{~Hz}$ for $x=0.95$. These values are somewhat higher than the $250 \mathrm{~Hz}$ found experimentally. It should be noted that Sato \& Kuriki found good agreement with their experimental values for Reynolds numbers based on the wake halfwidth, $b$, above 500. The same Reynolds number for the present case was about 100. This relatively low value of the Reynolds number indicates that perhaps viscous effects are not completely negligible. Undoubtedly more important, but presently unknown, is the influence of the decelerating external flow on the frequency. The analysis does, however, predict a considerably lower frequency than the $700 \mathrm{~Hz}$ primary wake frequency.

\section{Conclusions}

Throughout this study of vortex street breakdown we have taken the point of view that a certain distribution of vortices determines the velocity field of the wake. This idea was originated by von Kármán in his original summation of velocities induced by potential vortices. In the case of vortex street breakdown, we have found that it is necessary to extend the above approach by 
considering, in some detail, the convective processes acting on any given vortex.

The phenomenon of vortex street breakdown is characterized by the disappearance of a vortex street into a region where no significant velocity fluctuations are observed. This region, in turn, gives rise to another vortex street of larger scale and lower frequency than the original one. The detailed experimental findings suggest and are explained by a model in which a local concentration of vorticity, or vortex, is strained or convected by the other vortices of the street to an elliptical shape. This distorted vortex then rotates in the proper direction, through its self-induced velocities, approaching alignment of its major axis in the downstream direction. Because these processes take place as the vortices are travelling downstream, a region is reached where they touch or overlap to the extent they become shear layers on either side of the wake. These shear layers are then unstable and give rise to the secondary vortex street.

The convection of vorticity within a vortex by the other vortices of the street initiates the phenomenon. A spacing ratio greater than 0.366 is indicative of the straining of the vortices in the proper direction for the calm region to be formed. The reverse situation of straining such that the velocity fluctuations outside the street lag those inside $(h / a<0 \cdot 366)$ has, apparently, not been observed by other investigators and deserves further investigation.

It is interesting to speculate that similar processes may be present in turbulent flows. If small vortices making up a turbulent flow are subjected to the proper straining by other vortices a regrouping of vortices to a larger scale and hence lower frequency might occur.

The financial support of the National Science Foundation under NSF Grant GK-3854 is gratefully acknowledged.

\section{REFERENCES}

DURGIN, W. W. 1970 An experimental and analytical investigation of the mechanism causing the phenomenon of vortex street breakdown. Ph.D. thesis, Brown University.

HookER, S. G. 1936 On the action of viscosity in increasing the spacing ratio of a vortex street. Proc. Roy. Soc. A 154, 67-89.

Karmán, Th. von \& Rubach, H. 1912 Über den Mechanismus des Flussigkeits und Luftwider standes. Phys. Z. 13, Heft 2, 49-59.

Kovasznay, L. 1949 Hot-wire investigation of the wake behind cylinders at low Reynolds numbers. Proc. Roy. Soc. A 198, 175-190.

LAMB, H. 1945 Hydrodynamics, 6th edn. New York: Dover.

Payne, R. 1958 Calculations of unsteady viscous flow past a circular cylinder. J. Fluid Mech. 4, 81-86.

Roshko, A. 1953 On the development of turbulent wakes from vortex streets. NACA $T N 2913$.

SADEH, W., SUTERA, S. P. \& MAEder, P. F. 1968 An investigation of vorticity amplifica. tion in stagnation flow. Brown Univ., Div. of Engrg., Tech. Report WT-50.

SATO, H. \& KURIKI, K. 1961 The mechanism of transition in the wake of a thin flat plate placed parallel to a uniform flow. J. Fluid Mech. 11, 321-352.

SCHAFFER, J. W. \& Eskinazi, S. 1959 An analysis of the vortex street generated in 8 viscous fluid. J. Fluid Mech. 6, 241-260.

Taneda, S. 1959 Downstream development of the wakes behind cylinders. J. Phys. So. Japan, 14, 843-848.
On the phenomenon

TANEDA, S. 1965 Experimental investig $1714-1721$.

Wood, R. T. 1969 An experimental and lying the influence of free-stream $t$ from cylinders in cross flow. Ph.D. $t$

ZDRAVKovich, M. 1968 Smoke observa at low Reynolds number. $J$. Fluid $N$ 


\section{F. Karlsson}

cesses acting on any given vortex. own is characterized by the disere no significant velocity fluctuais rise to another vortex street of nal one. The detailed experimental 1 in which a local concentration of by the other vortices of the street ien rotates in the proper direction, ing alignment of its major axis in :esses take place as the vortices are here they touch or overlap to the de of the wake. These shear layers ury vortex street.

: by the other vortices of the street ater than 0.366 is indicative of the $n$ for the calm region to be formed. 1e velocity fluctuations outside the rently, not been observed by other ion.

'ocesses may be present in turbulent it flow are subjected to the proper ortices to a larger scale and hence

nce Foundation under NSF Grant

\section{ES}

lytical investigation of the mechanism zdown. Ph.D. thesis, Brown University. increasing the spacing ratio of a vortex len Mechanismus des Flussigkeits und ). wake behind cylinders at low Reynolds Jrk: Dover.

s flow past a circular cylinder. J. Fluid lent wakes from vortex streets. $N A C A$

An investigation of vorticity amplificaEngrg., Tech. Report WT-50.

of transition in the wake of a thin flat uid Mech. 11, 321-352.

isis of the vortex street generated in a le wakes behind cylinders. J. Phys. Soc.
On the phenomenon of vortex street breakdown

TANEDA, S. 1965 Experimental investigation of vortex streets. J. Phys. Soc. Japan, 20, 1714-1721.

Wood, R. T. 1969 An experimental and analytical investigation of the mechanism underlying the influence of free-stream turbulence on the stagnation line heat transfer from eylinders in cross flow. Ph.D. thesis, Brown University.

ZDRAVkovich, M. 1968 Smoke observations of the wake of a group of three cylinders at low Reynolds number. J. Fluid Mech. 32, 339-351. 